> Les interfaces que vivent quotidiennement les praticiens et les chercheurs à propos des ressources biologiques humaines pour le cancer sont à la fois compliquées et en constante évolution. <

\section{Contractualisation des relations entre biobanques et chercheurs}

\author{
Les material transfer \\ agreements (MTA)
}

Anne Janin

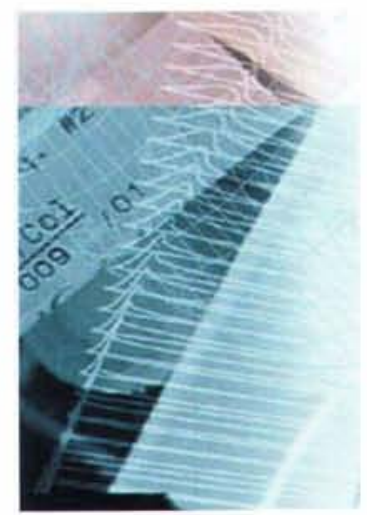

Cancéropole lle-de-France,

Laboratoire de Pathologie et

Inserm U.728-Université Paris 7,

Hôpital Saint-Louis,

1, avenue Claude Vellefaux,

75010 Paris, France.

janin@chu-stlouis.fr.
La mise en application des nouvelles lois comme les demandes de valorisation rendent nécessaire la mise en place, par les institutions partenaires pour le soin et la recherche, de documents réglant les conditions d'échange de ces ressources biologiques humaines. Le terme anglo-saxon qui les désigne est material transfer agreement (MTA).

\section{Les éléments constants d'un MTA}

Schématiquement, le document réglant les échanges de matériel biologique humain doit comprendre dans tous les cas:

\section{La description précise du type de matériel} biologique humain qui est transmis : cellules, tissus, acides nucléiques, protéines Il apparaît en pratique très important de donner des éléments garantissant la qualité du matériel transmis. Déterminer précisément la quantité de matériel transmis est tout aussi important, car la question des reliquats restant à la fin du programme de recherche doit être évoquée. L'usage de ces reliquats pour un autre programme de recherche que le programme pour lequel le malade a été informé et/ou a donné son accord doit en effet être proscrit. Déterminer avec le partenaire utilisateur du matériel biologique la quantité de matériel nécessaire à la réalisation de son programme apparaît donc nécessaire pour éviter d'engendrer des reliquats.

L'identification des partenaires

II s'agit des «donneurs » et des « receveurs » entre lesquels la transmission de matériel biologique a lieu. Ces partenaires clairement identifiés seront responsables en cas de plainte.

La finalité de l'échange

Elle comporte la description précise du programme de recherche pour lequel la transmission de matériel biologique humain a lieu.

En effet, il est essentiel que le matériel biologique humain qui est transmis entre les deux partenaires du MTA soit bien utilisé pour un programme de recherche donné et seulement pour ce programme de recherche (Figure 1). 


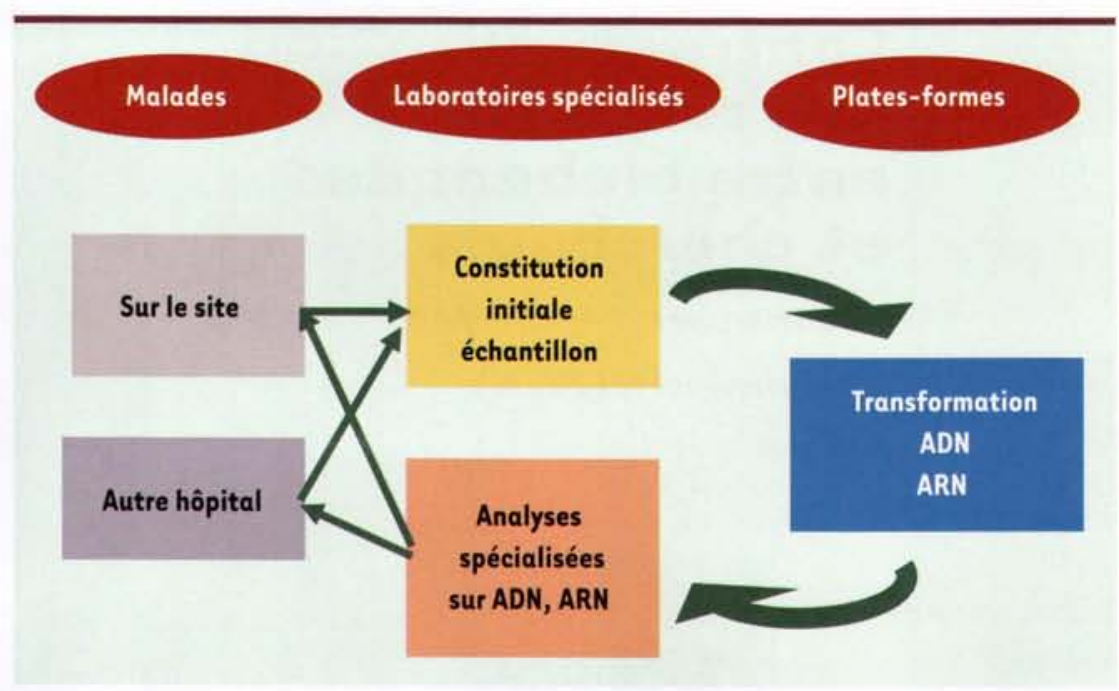

Figure 1. Le circuit diagnostique.

\section{Les éléments variables du MTA}

Les données économiques qui sont autour de cet échange sont, elles, très variées.La rédaction, le contrôle et le suivi de ces documents doivent donc être pris en charge par les institutions, tant hospitalières que de recherche. La mise à disposition de matériel biologique humain peut se faire en demandant le remboursement des frais de préparation de ce matériel biologique (Figure 2). Elle peut aussi s'accompagner de clauses de valorisation.

En effet, la mise à disposition de matériel biologique humain pour la recherche s'accompagne souvent de mise à disposition d'annotations cliniques et/ou biologiques résultant du travail des médecins et biologistes qui ont pris en charge le malade. La définition du droit de propriété intellectuelle de ces médecins et biologistes sur les découvertes qui peuvent découler du programme de recherche utilisant le matériel

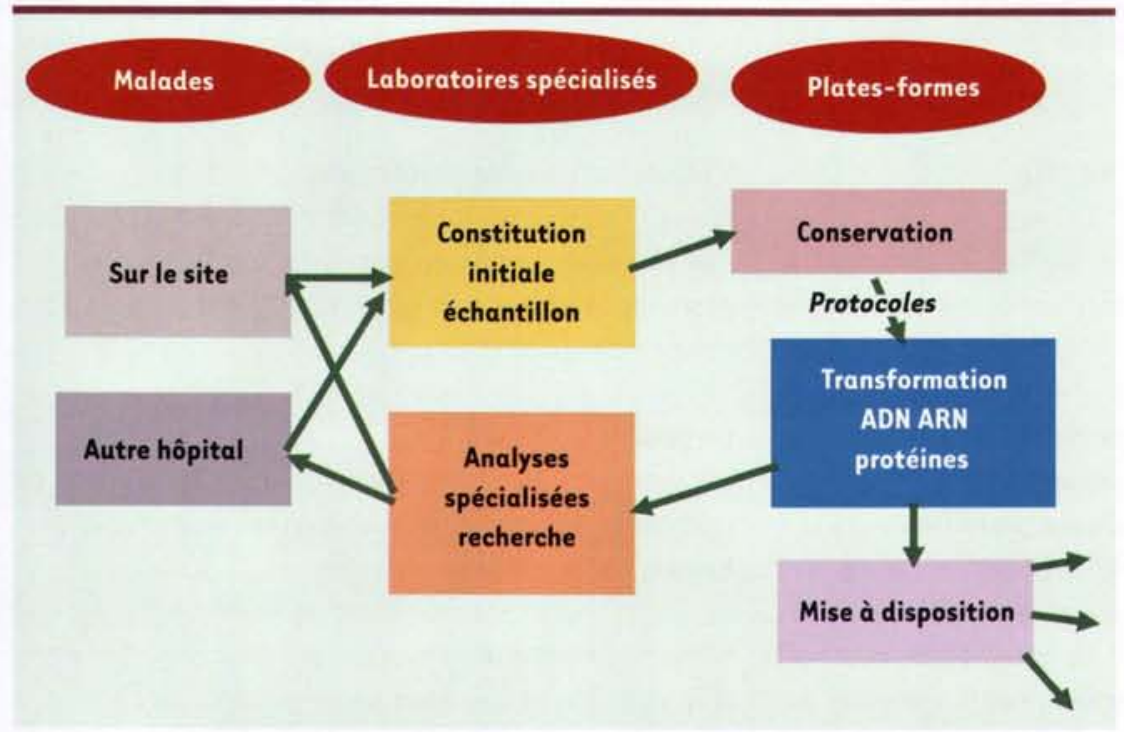

Figure 2. Le circuit recherche-valorisation. biologique transmis avec ses annotations est étudiée encore actuellement au cas par cas, et reste difficile à définir comme à négocier.

\section{Conclusion}

Les MTA permettent donc donner un cadre réglementaire aux échanges de matériel biologique humain entre biobanques et chercheurs. Dans les conditions actuelles, il est recommandé de les établir et de les suivre au niveau institutionnel. Une pratique plus régulière des échanges permettra sans doute d'harmoniser les règles de valorisation encore très discutées dans ce domaine. $\diamond$

\section{SUMMARY}

Contractual relationships

between biobanks and researchers, the material transfer agreements

Daily interfaces actual experiences by practitioners and researchers about human biological resources for cancer are at the same time complicated and constantly evolved. $\nabla$
TIRÉS À PART

A. Janin 